\title{
Um Modelo de Otimização Multiobjetivo para Análise de Sistema de Recursos Hídricos I: Metodologia
}

\author{
Valterlin da Silva Santos, Wilson Fadlo Curi, Rosires Catão Curi, Allan Sarmento Vieira \\ Universidade Federal de Campina Grande \\ valterlin@yahoo.com.br; wfcuri@yahoo.com.br; rosirescuri@yahoo.com.br; allansarmento@yahoo.com.br
}

Recebido: 14/01/10 - revisado: 27/05/10 - aceito: 26/08/11

\section{RESUMO}

Este trabalho apresenta um modelo de otimização multiobjetivo, baseado em programação linear, para o estudo integrado da operação de sistemas de reservatórios e perímetros irrigados, além de outros usos da água, estabelecendo a alocação ótima dos recursos naturais existentes (água e terras aptas ao plantio) para o planejamento e estabelecimento de políticas operacionais ótimas em bacias hidrográficas. Os múltiplos usos da água incluem o atendimento de demandas via reservatórios ou a fio d'água, como de irrigação ou de demandas urbanas, controle de cheias, regularização de vazões, prevenção contra as secas e volumes metas dos reservatórios. Aspectos econômicos e sociais, como a maximização da receita líquida e da geração de empregos oriunda da agricultura irrigada, são contemplados pelo modelo. As não-linearidades das funções objetivo e dos processos representados por cada restrição foram implementadas através do uso combinado de técnicas matemáticas de linearizações.

Palavras - chave: Recursos hídricos, otimização, programação linear, linearização.

\section{INTRODUÇÃOO}

O planejamento e gerenciamento dos recursos hídricos têm importância fundamental no estabelecimento de diretrizes e ações para o aproveitamento, controle e conservação desses recursos, principalmente em regiões onde há escassez hídrica.

Por ser um problema de grande complexidade, a alocação de água entre os usos múltiplos pode ser solucionada através de técnicas de análise de sistemas numa abordagem sistêmica e do uso de técnicas computacionais agregadas à modelagem matemática.

A formulação matemática busca descrever a dinâmica do sistema pela utilização de vários tipos de equações matemáticas, geralmente recorrendo a equação do balanço hídrico, na forma de restrições de igualdade, que traduz o princípio de conservação da massa. Associados a essa dinâmica são incorporadas as expressões limitantes do sistema, normalmente na forma de restrições de desigualdade (físicas, operacionais, condicionais, etc.), e as funções objetivo, que estabelecem a forma de valoração dos resultados do processo decisório.

Quando existe o interesse em desenvolver um planejamento para valorar e procurar otimizar certos aspectos do processo decisório, devem-se aplicar técnicas de otimização. Uma técnica que merece destaque é a Programação Linear (PL), por determinar o ótimo global, se ele existir, além de ser uma técnica bem estabelecida para a qual há diversos softwares disponíveis. Ela foi estabelecida por G. B. Dantzig em 1947, para resolver problemas de logística da Força Área Americana.

A principal desvantagem da PL é a hipótese da linearidade das relações entre as variáveis. Porém, sob certas hipóteses, problemas não-lineares podem ser linearizados utilizando-se de aproximações matemáticas ou de procedimentos iterativos. Entretanto, a maioria das metodologias existentes (Barbosa, 2002; Lanna, 1998) apenas linearizam a função objetivo do modelo. Normalmente as nãolinearidades intrínsecas aos processos de cada componente, representadas nas equações de restrição do modelo, como as curvas cota-área-volume, por exemplo, não são consideradas no modelo (Hsu et al., 2008; Mousavi et al., 2004) ou são linearizadas com o uso de regressão linear simples (Sudha et al., 2008; Diaz et al., 2000). Além do mais, muitos trabalhos utilizam apenas uma função objetivo (Sudha et al., 2008; Ahmed e Sarma, 2005; Curi et al., 2004) para analisar o sistema, não contemplando, simultaneamente, diferentes formas de avaliação do uso dos recursos hídricos durante um processo decisório. 
Contudo, este trabalho apresenta um modelo de otimização, baseado em programação linear, para prover o estudo da alocação ótima dos recursos naturais existentes (água e terras aptas ao plantio) entre os múltiplos usos de um sistema de recursos hídricos, quando operado de forma integrada, via uma análise multiobjetivo.

Pretende-se estabelecer uma metodologia capaz de ser aplicado a sistemas de recursos hídricos, com a possibilidade de uma representação mais detalhada do sistema, tendo em vista a escolha de políticas operacionais que possam melhorar de forma sustentável, o uso da água e das áreas irrigadas em regiões com escassez hídrica, minimizando problemas peculiares de déficit hídrico e os conflitos de uso da água.

\section{MODELO DE OTIMIZAÇÃO}

O modelo proposto destina-se a otimizar os múltiplos usos de um sistema de recursos hídricos, com a implantação ou melhoramento da operação de um ou mais perímetros irrigados.

A representação do sistema (Figura 1) é constituída por elementos artificiais, como nós e links, e naturais, descritos a seguir:

- Nó: elemento artificial onde se interligam dois ou mais elementos do sistema.

- Link: elemento artificial utilizado para representar uma ligação entre os elementos do sistema. Pode ser um trecho do rio, canal, adutora ou uma tomada d'água (estrutura hidráulica para derivar água para atender alguma demanda hídrica, cuja vazão é independente da altura da coluna d'água na fonte).

- Afluência: representa a vazão de entrada ao sistema oriunda de alguma fonte de água. Pode estar conectado a um nó ou a um reservatório.

- Reservatório: elemento destinado a armazenar água. Ele pode estar conectado a uma afluência, a um descarregador de fundo, a um vertedouro ou a tomadas d'água.

- Vertedouro: canal artificial,conectado ao reservatório e a um nó do sistema, com a finalidade de conduzir, de forma segura, o excesso de água armazenado no reservatório.

- Descarregador de fundo: elemento utilizado para descarga de água de um reservatório, cuja vazão máxima é controlada pela diferença entre o nível de água no reservatório e no leito do rio localizado em sua saída. Está conectado ao reservatório e a um nó do sistema.

- Demanda: quantidade de água requerida por determinado usuário do sistema. Pode se conectar a um nó ou a um reservatório.

- Perímetro irrigado: área destinada ao cultivo de culturas agrícolas com infra-estrutura e procedimentos capazes de permitir a aplicação da água nas culturas agrícolas. Pode se conectar a um nó ou a um reservatório.

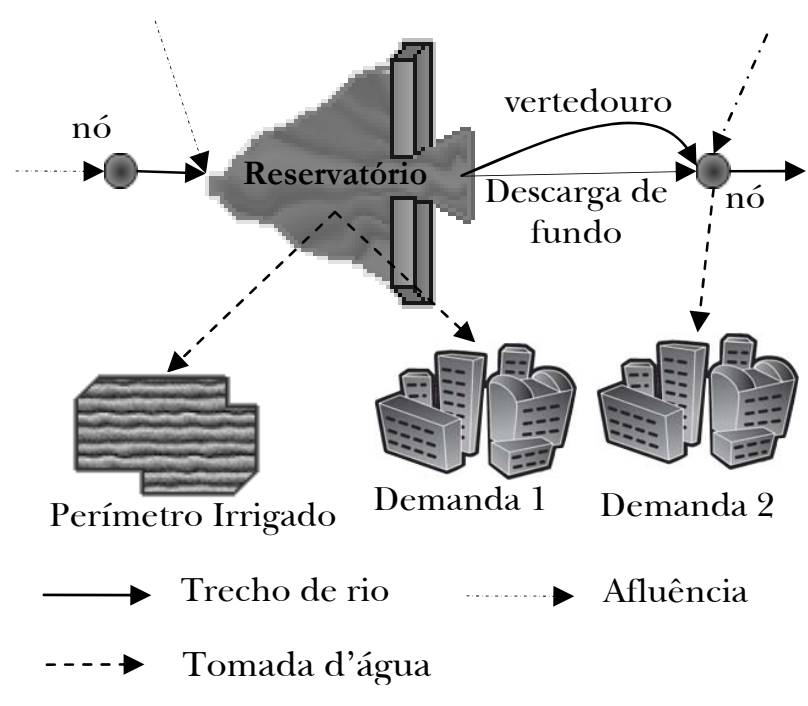

Figura 1 - Componentes para representação do sistema

As variáveis do modelo e as equações que regem o seu comportamento estão relacionadas com as informações obtidas dos elementos naturais (como aspectos hidroclimáticos, hidroagrícolas, etc.) e da infraestrutura (das demandas hídricas, das características físicas dos componentes hidráulicos das estruturas de armazenamento e transporte de água) identificadas no sistema hídrico em estudo. A dinâmica de operação do reservatório e dos nós é fundamentada na equação do balanço hídrico. A demanda hídrica de cada perímetro irrigado é determinada com base na necessidade suplementar líquida de irrigação, estabelecidas através do balanço hídrico no solo para as culturas selecionadas, estando a área a ser plantada limitada pela disponibilidade de água e solo, além da sua prioridade de atendimento. Também é levado em consideração os diferentes tipos de sistemas de irrigação, suas necessidades de altura manométrica, as áreas a serem 
irrigadas para cada tipo de cultura agrícola, preço da água e de produção, os aspectos econômicos e a combinação ou variação nas fontes de bombeamento e a quantidade de água captada.

O modelo desenvolvido utiliza o Toolbox Optimization do software MATLAB 6.5 com o Método do Ponto Interior para a busca da solução ótima. A função objetivo é uma combinação linear das funções objetivo do problema, ou seja, utilizando o Método das Ponderações (Braga e Gobetti, 2002). Cada função objetivo é normalizada e as ponderações são pesos atribuídos às funções para definir suas prioridades de atendimento. Tais funções estão sujeitas a inúmeras restrições, representadas por equações de natureza linear e não linear, que traduzem, a nível mensal, as limitações físicas dos reservatórios, perímetros irrigados e equipamentos hidráulicos, e limitações hidrológicas, legais, econômicas e sociais, inerentes aos sistemas de usos múltiplos. As não-linearidades das funções objetivo e dos processos representados em cada restrição foram implementadas através do uso combinado de técnicas matemáticas de linearizações, denominadas Artifício de Linearização por Segmentos (Lanna, 1998) e Programação Linear Seqüencial ou Sucessiva (Barbosa, 2002).

As equações matemáticas representativas das funções objetivo e restrições serão abordadas na seqüência.

\section{Função objetivo}

O modelo proposto apresenta 5 (cinco) funções objetivo, que serão tratadas a seguir.

A Equação 1 representa a função objetivo destinada à minimização do déficit do atendimento das demandas nas tomadas d'água dos reservatórios e dos nós do sistema.

$\operatorname{DTD}_{\mathrm{d}}=\sum_{\mathrm{t}}\left(\frac{\mathrm{Dt}_{\mathrm{dt}}-\mathrm{Qt}_{\mathrm{dt}}}{\mathrm{Dt} \mathrm{t}_{\mathrm{dt}}}\right)^{2}$

sendo d a d-ézima tomada d'água do sistema; $\mathrm{Dt}_{\mathrm{dt}}$ a demanda requerida na tomada d'água d no mês t; e $\mathrm{Qt}_{\mathrm{dt}}$ o volume mensal destinado ao atendimento da demanda na tomada d'água d no mês t $\left(\mathrm{Qt}_{\mathrm{dt}} \leq \mathrm{Dt}_{\mathrm{dt}}\right)$.

Para que a Equação 1 possa ser utilizada em PL, optou-se por linearizá-la através do Artifício de Linearização por Segmentos, dividindo a vazão destinada ao atendimento da demanda na tomada d'água, no mês t, em 4 segmentos iguais, como mostra a Figura 2.
Assim a função objetivo quadrática e o volume mensal destinado ao atendimento das tomadas d'água do sistema serão representados por:

$$
\begin{aligned}
& \left(\frac{\mathrm{Dt}_{\mathrm{dt}}-\mathrm{Qt}_{\mathrm{dt}}}{\mathrm{Dt} \mathrm{t}_{\mathrm{dt}}}\right)^{2}=1+\sum_{\mathrm{q}=1}^{4} \phi \mathrm{t}_{\mathrm{dq}} \cdot \mathrm{qt}_{\mathrm{dqt}} \\
& \mathrm{Qt}_{\mathrm{dt}}=\sum_{\mathrm{q}=1}^{4} \mathrm{qt}_{\mathrm{dqt}}
\end{aligned}
$$

sendo $\mathrm{qt}_{\mathrm{dqt}}$ os segmentos de volumes mensais destinados ao atendimento da demanda na tomada d'água $\mathrm{d}$ no mês $\mathrm{t}, \mathrm{q}=1, \ldots, 4 ; \phi \mathrm{t}_{\mathrm{dq}}$ a declividade do segmento de reta $q$ da função objetivo destinada ao atendimento da demanda na tomada d'água $\mathrm{d}$; $\mathrm{qt}_{\mathrm{dqt}}$ o incremento do volume mensal destinado ao atendimento da demanda na tomada d'água d do trecho segmentado q, no mês t, limitado por:

$0 \leq \mathrm{qt}_{\mathrm{dqt}} \leq 0,25 \cdot \mathrm{Dt}_{\mathrm{dt}}$

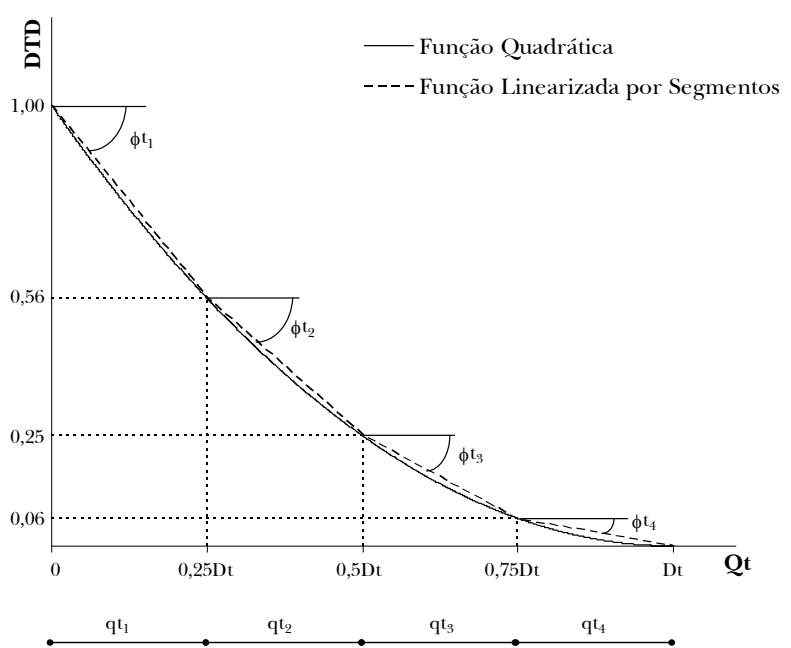

Figura 2 - Gráfico da função objetivo quadrática linearizada por segmentos da demanda de uma tomada d'água d para um mês t.

O volume mensal defluente dos reservatórios permite a perenização ou regularização da vazão a jusante, assim como o atendimento das demandas ecológicas ou outros usos da água. A Equação 5 representa a função objetivo que se destina a minimizar o déficit entre o volume defluente liberado pelo reservatório $r$ no mês $\mathrm{t}\left(\mathrm{Qe}_{\mathrm{rt}}\right)$ e a demanda mínima a ser mantida a jusante do reservatório $\mathrm{r}$ no mês t $\left(\mathrm{De}_{\mathrm{rt}}\right)$ : 
$\mathrm{VE}_{\mathrm{r}}=\sum_{\mathrm{t}}\left|\frac{\mathrm{De}_{\mathrm{rt}}-\mathrm{Qe}_{\mathrm{rt}}}{\mathrm{De}_{\mathrm{rt}}}\right|$

O volume meta se destina a manter o volume de água do reservatório em certo nível para atender certas demandas, como controle de cheias, recreação, piscicultura, ou geração de energia elétrica. O modelo tem como objetivo minimizar o déficit entre o volume de água do reservatório $\mathrm{r}$ no final do mês $t\left(\mathrm{VR}_{\mathrm{rt}}\right)$ e o volume meta estabelecido neste mês (VRmeta $\left.{ }_{\mathrm{rt}}\right)$, representada na Equação 6:

$\mathrm{VM}_{\mathrm{r}}=\sum_{\mathrm{t}}\left(\frac{\text { VRmeta }_{\mathrm{rt}}-\mathrm{VR}_{\mathrm{rt}}}{\text { VRmeta }_{\mathrm{rt}}}\right)^{2}$

Logo a Equação 6 será linearizada através do Artifício de Linearização por Segmentos. Um possível exemplo de linearização da equação 6 é mostrado na Figura 3.

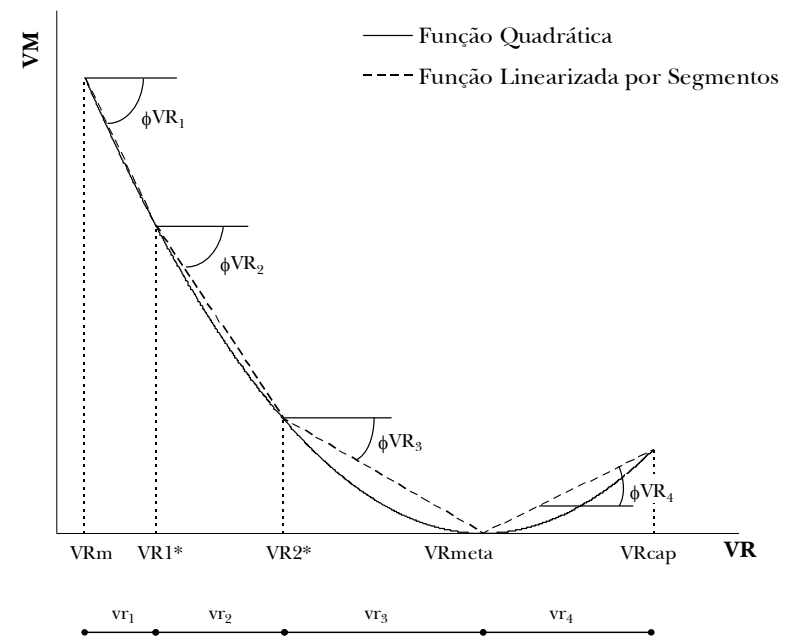

Figura 3 - Exemplo de um gráfico da função objetivo quadrática linearizada por segmentos do volume meta de um reservatório $r$ para um mês $t$.

O volume morto, VRm, e a capacidade do reservatório, VRcap, assim como os volumes intermediários, VR1* e VR2*, são volumes de controle (ou pontos de quebra) que devem ser definidos previamente. Cada trecho segmentado $\mathrm{m}$ do volume de água do reservatório $\mathrm{r}$ esta limitado pelos volumes de controle.

Assim a função objetivo quadrática para o volume meta e o volume de água do reservatório $r$, em um dado mês t, será representada por:
$\left(\frac{\mathrm{VRmeta}_{\mathrm{rt}}-\mathrm{VR}_{\mathrm{rt}}}{\text { VRmeta }_{\mathrm{rt}}}\right)^{2}=\mathrm{a}_{\mathrm{rt}}+\sum_{\mathrm{m}=1}^{\mathrm{nm}} \phi \mathrm{VR}_{\mathrm{rmt}} \cdot \mathrm{vr}_{\mathrm{rmt}}$

$\mathrm{VR}_{\mathrm{rt}}=\mathrm{VRm}_{\mathrm{r}}+\sum_{\mathrm{m}=1}^{\mathrm{nm}} \mathrm{vr}_{\mathrm{rmt}}$

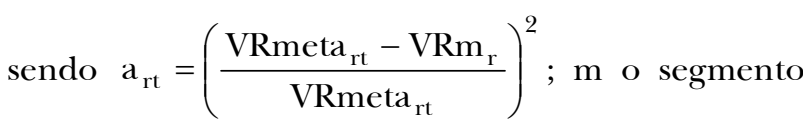
de cada trecho do volume de água do reservatório $r$ no mês $\mathrm{t}, \mathrm{m}=1, \ldots, \mathrm{nm},(\mathrm{nm}=$ número de segmentos do volume de água do reservatório); $\phi \mathrm{VR}_{\mathrm{mt}} \mathrm{a}$ declividade do segmento de reta $\mathrm{m}$ da função objetivo do volume meta do reservatório $\mathrm{r}$ no mês $\mathrm{t}$; $\mathrm{vr}_{\mathrm{mt}}$ o incremento do volume de água do reservatório $r$ para o trecho segmentado $\mathrm{m}$, no mês t.

Os volumes VR1*, VR2, podem ser volumes físicos pré-estabelecidos, como volume corresponde a cota de entrada do descarregador de fundo ou da cota de entrada da tomada d'água, bem como pontos da curva cota-área-volume.

Outra função objetivo do modelo é a maximização da receita líquida (RL) oriunda da agricultura irrigada, entendida como o resultado da diferença entre a renda bruta total auferida com a venda da safra agrícola e os respectivos custos de produção envolvidos, gerada pela escolha apropriada das áreas a serem irrigadas para cada tipo de cultura agrícola prevista nos perímetros irrigados.

Portanto, a receita líquida de um perímetro irrigado $\mathrm{k}$, em ( $\mathrm{R} \$ / \mathrm{ano})$ é dada pela expressão:

$\mathrm{RL}_{\mathrm{k}}=\sum_{\mathrm{n}=1 \mathrm{j}=1}^{\mathrm{na}} \sum_{\mathrm{nc}}^{\mathrm{Rc}} \mathrm{b}_{\mathrm{kjn}}-\mathrm{Cp}_{\mathrm{kjn}}-\mathrm{Ca}_{\mathrm{kjn}}-\mathrm{Cb}_{\mathrm{kjn}}$

sendo $\mathrm{n}$ o ano, $\mathrm{n}=1,2, \ldots$, na, $(\mathrm{na}=$ número de $\mathrm{a}-$ nos); $\mathrm{j}$ o tipo de cultura agrícola, $\mathrm{j}=1,2, \ldots, \mathrm{nc},(\mathrm{nc}=$ número de culturas); $\mathrm{Rb}_{\mathrm{kjn}}$ a renda bruta anual da cultura agrícola $\mathrm{j}$ no ano $\mathrm{n}$ no perímetro irrigado $\mathrm{k}$; $\mathrm{Cp}_{\mathrm{kjn}}$ o custo de produção anual da cultura agrícola $\mathrm{j}$ no ano $\mathrm{n}$ no perímetro irrigado $\mathrm{k}$; $\mathrm{Ca}_{\mathrm{kjn}} \mathrm{o}$ custo da água anual usada na cultura agrícola $\mathrm{j}$ no ano $\mathrm{n}$ no perímetro irrigado $\mathrm{k}$; $\mathrm{Cb}_{\mathrm{kjn}}$ o custo anual de bombeamento de água para a cultura agrícola j no ano $\mathrm{n}$ no perímetro irrigado $\mathrm{k}$;

A renda bruta anual no perímetro irrigado $\mathrm{k}$ $\left(R b_{\text {kjn }}\right)$, em $\mathrm{R} \$ /$ ano/cultura, pode ser estimada por:

$\operatorname{Rb}_{\mathrm{kjn}}=\operatorname{Prod}_{\mathrm{kjn}} \cdot \operatorname{Prc}_{\mathrm{jn}} \cdot \mathrm{Ac}_{\mathrm{kjn}}$

sendo $\operatorname{Prod}_{\mathrm{kjn}}$ a produtividade da cultura agrícola $\mathrm{j}$ por unidade de área no perímetro irrigado $\mathrm{k}$ no ano 
n; $\operatorname{Prc}_{\mathrm{jn}}$ é o preço atual de comercialização da cultura agrícola j no ano $n$ e $\mathrm{Ac}_{\mathrm{kjn}}$ é a área plantada com a cultura agrícola j no perímetro irrigado k no ano n.

O custo de produção anual no perímetro irrigado $\mathrm{k}\left(\mathrm{Cp}_{\mathrm{kjn}}\right)$, em $\mathrm{R} \$ /$ ano/cultura, relativos aos gastos com insumos, mão de obra e máquinas, entre outros, pode ser dado por:

$\mathrm{Cp}_{\mathrm{kjn}}=\operatorname{Cprod}_{\mathrm{kjn}} \cdot \mathrm{Ac}_{\mathrm{kjn}}$

sendo $\operatorname{Cprod}_{\text {kjn }}$ o custo atual de produção por unidade de área da cultura agrícola j referentes aos gastos relativos ao ano $\mathrm{n}$ no perímetro irrigado $\mathrm{k}$.

$\mathrm{O}$ custo da água anual, $\mathrm{Ca}_{\mathrm{kjn}} \quad(\mathrm{em}$ $\mathrm{R} \$$ /ano/cultura), captada para o perímetro irrigado k pode ser obtido pela expressão:

$\mathrm{Ca}_{\mathrm{kjn}}=\sum_{\mathrm{t}=12(\mathrm{n}-1)+1}^{12(\mathrm{n}-1)+12} \operatorname{Pra}_{\mathrm{k}} \cdot \operatorname{Qirr}_{\mathrm{kjt}} \cdot \mathrm{Ac}_{\mathrm{kjn}}$

sendo $\operatorname{Pra}_{\mathrm{k}}$ é o preço da água por unidade de volume destinado ao perímetro irrigado $\mathrm{k}$ e Qirr $_{\mathrm{jkt}}$ é a lâmina mensal de água para a irrigação da cultura agrícola j no perímetro irrigado $\mathrm{k}$ durante o mês $\mathrm{t}$, transformada em vazão por unidade de área fornecida pelo sistema, obtida por:

$\operatorname{Qirr}_{\mathrm{kjt}}=\frac{\mathrm{Nl}_{\mathrm{kjt}}}{\left(1-\mathrm{LR}_{\mathrm{kjt}}\right) \cdot \mathrm{Esis}_{\mathrm{kj}} \cdot \mathrm{Eap}_{\mathrm{j}}}$

sendo $\mathrm{Nl}_{\mathrm{kjt}}$ a necessidade hídrica suplementar da cultura agrícola $\mathrm{j}$ no perímetro irrigado $\mathrm{k}$ durante $\mathrm{o}$ mês t; $\mathrm{LR}_{\mathrm{kft}}$ é a necessidade de lixiviação dos sais para a cultura agrícola $\mathrm{j}$ no mês t no perímetro irrigado k; Esis ${ }_{\mathrm{kj}}$ é a eficiência do sistema de distribuição de água para cada cultura agrícola j no perímetro irrigado k e $\operatorname{Eap}_{\mathrm{j}}$ é a eficiência da aplicação da irrigação por cultura agrícola.

A necessidade hídrica suplementar $\left(\mathrm{Nl}_{\mathrm{kjt}}\right)$ ou a lâmina de rega suplementar que a planta necessita, para cada intervalo de tempo do seu ciclo vegetativo, pode ser estimada por:

$\mathrm{Nl}_{\mathrm{kjt}}=\mathrm{ETP}_{\mathrm{kjt}}-\mathrm{Pe}_{\mathrm{kt}}-\mathrm{G}_{\mathrm{kjt}}-\mathrm{W}_{\mathrm{kt}}$

sendo $\mathrm{Pe}_{\mathrm{kt}}$ a taxa de precipitação que infiltra no solo, que fica efetivamente a disposição das plantas no mês t no perímetro irrigado k; $G_{\mathrm{kjt}}$ é a dotação de água à zona radicular da cultura $\mathrm{j}$ durante o mês $\mathrm{t}$ por capilaridade que depende do tipo de solo e do nível do lençol freático no perímetro irrigado $\mathrm{k} e$ $\mathrm{W}_{\mathrm{kt}}$ é a reserva de água no solo no inicio do mês $\mathrm{t}$ que depende da capacidade de armazenamento de água no solo na unidade de produção ou perímetro irrigado k. $\mathrm{ETP}_{\mathrm{kjt}}$ é a taxa de evapotranspiração potencial da cultura agrícola j no perímetro irrigado $\mathrm{k}$ durante o mês $t$, que pode ser estimada, de forma aproximada, por:

$\mathrm{ETP}_{\mathrm{kjt}}=\mathrm{kc}_{\mathrm{jt}} \cdot \mathrm{ETO}_{\mathrm{kt}}$

sendo $\mathrm{kc}_{\mathrm{jt}}$ é o coeficiente de cultivo mensal da cultura agrícola j que reflete a sua necessidade hídrica no mês t e $\mathrm{ETO}_{\mathrm{kt}}$ é a taxa de evaporação de referência no mês t no perímetro irrigado $\mathrm{k}$.

O custo anual de bombeamento de água, $\mathrm{Cb}_{\mathrm{jn}}$ (em $\mathrm{R} \$ /$ ano/cultura) para as áreas irrigadas é obtido através da expressão:

$\mathrm{Cb}_{\mathrm{kjn}}=\sum_{\mathrm{t}=12(\mathrm{n}-1)+1}^{12(\mathrm{n}-1)+12}\left(\frac{\mathrm{b}_{\mathrm{kjt}}}{\eta_{\mathrm{k}}}\right)$

sendo $b_{\mathrm{kjt}}=0,02726 \cdot \operatorname{Prb}_{\mathrm{k}} \cdot \Delta \mathrm{H}_{\mathrm{kjt}} \cdot \operatorname{Qirr}_{\mathrm{kjt}} \cdot \mathrm{Ac}_{\mathrm{kjn}} ; \operatorname{Prb}_{\mathrm{k}}$ o preço da energia elétrica (em $\mathrm{R} \$ / \mathrm{Kwh}$ ) para o perímetro irrigado $\mathrm{k} ; \Delta \mathrm{H}_{\mathrm{kjt}}$ é a altura manométrica média (em metros de coluna de água), requerido pelo sistema de irrigação da cultura agrícola j e aduzido para o perímetro irrigado $k$ no mês $t$ e $\eta_{k}$ é a eficiência do sistema de bombeamento do perímetro irrigado $\mathrm{k}$.

A maximização da mão-de-obra oriunda da atividade agrícola nos perímetros irrigados é outra função objetivo sujeita às mesmas restrições agronômicas. A mão-de-obra total empregada MO (em diárias/ano) requerida nas unidades de produção ou perímetros é dada pela expressão:

$\mathrm{MO}_{\mathrm{k}}=\sum_{\mathrm{n}=1 \mathrm{j}=1}^{\mathrm{na}} \sum_{\mathrm{nc}}^{\mathrm{nc}} \mathrm{Hdc}_{\mathrm{kj}} \cdot \mathrm{Ac}_{\mathrm{kjn}}$

sendo $\mathrm{Hdc}_{\mathrm{kj}}$ a mão-de-obra, por unidade de área, requerida pela cultura agrícola $\mathrm{j}$ no perímetro irrigado k;

O modelo utiliza o Método das Ponderações para efetuar a análise multiobjetivo, sendo cada função objetivo, descrita anteriormente, normalizada. Logo, a função objetivo do modelo é dada pela Equação 18.

$$
\begin{aligned}
\min \text { fo }= & \sum_{\mathrm{d}} \omega_{1 \mathrm{~d}} \cdot \mathrm{DTD}_{\mathrm{d}}+\sum_{\mathrm{r}} \omega_{2 \mathrm{r}} \cdot \mathrm{VE}_{\mathrm{r}}+ \\
& \sum_{\mathrm{r}} \omega_{3 \mathrm{r}} \cdot \mathrm{VM}_{\mathrm{r}}+\sum_{\mathrm{k}} \omega_{4_{\mathrm{k}}} \cdot \mathrm{RL}_{\mathrm{k}}+ \\
& \sum_{\mathrm{k}} \omega_{5 \mathrm{k}} \cdot \mathrm{MO}_{\mathrm{k}}
\end{aligned}
$$


sendo $\mathrm{d}$, r e k, respectivamente, os índices para representar as demandas, os reservatórios e os perímetros irrigados e $\omega_{*}$ o coeficiente de ponderação que mede a relativa importância ou prioridade de atendimento entre as funções objetivo. Quando $\omega_{*}=0 \mathrm{a}$ função objetivo não será considerada no processo de otimização.

\section{Equações de restrição}

Os reservatórios podem ser conectados a outros elementos do sistema como a nós, descargas de fundo, vertedouros e tomadas de água (Figura 4), tendo o balanço hídrico, baseado no princípio de conservação da massa, expressa pela Equação 19.

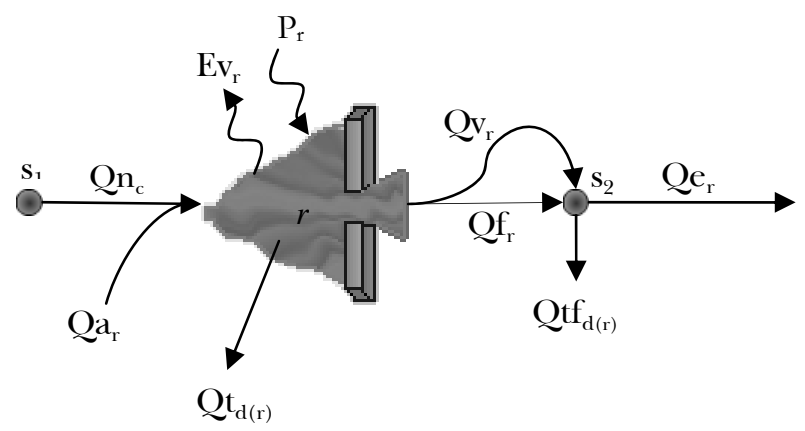

Figura 4 - Diagrama representando os componentes do reservatório avaliados pelo modelo.

$$
\begin{aligned}
\mathrm{VR}_{\mathrm{rt}}= & \mathrm{VR}_{\mathrm{r}(\mathrm{t}-1)}+\mathrm{Qa}_{\mathrm{rt}}-\sum_{\mathrm{d}(\mathrm{r})} \mathrm{Qtr}_{\mathrm{d}(\mathrm{r}) \mathrm{t}}-\mathrm{Qf}_{\mathrm{rt}} \\
& -\mathrm{Qv}_{\mathrm{rt}}+\mathrm{P}_{\mathrm{rt}}-\mathrm{Ev}_{\mathrm{rt}}+\mathrm{Qn}_{\mathrm{ct}}
\end{aligned}
$$

sendo s o índice que representa o s-ézimo nó do sistema; $\mathrm{VR}_{\mathrm{rt}}$ o volume do reservatório $\mathrm{r}$ no final do mês $t ; \mathrm{VR}_{\mathrm{r}(\mathrm{t}-1)} \mathrm{o}$ volume do reservatório $\mathrm{r}$ no inicio do mês $\mathrm{t} ; \mathrm{Qa}_{\mathrm{rt}}$ a volume afluente ao reservatório $\mathrm{r}$ durante o mês t; $d(r)$ o índice que representa a $d-$ ézima tomada d'água do reservatório $r ; \mathrm{Qt}_{\mathrm{d}(\mathrm{r}) \mathrm{t}} \mathrm{o}$ dézimo volume alocado para a tomada d'água do reservatório $\mathrm{r}$ no mês $\mathrm{t}$; $\mathrm{Qtf}_{\mathrm{d}(\mathrm{r}) \mathrm{t}}$ o d-ézimo volume alocado para a tomada d'água de fundo do reservatório $\mathrm{r}$ no mês t; $\mathrm{Qf}_{\mathrm{rt}} \mathrm{o}$ volume liberado através da descarga de fundo do reservatório $r$ durante o mês t; $\mathrm{Qv}_{\mathrm{rt}}$ o volume vertido do reservatório $\mathrm{r}$ durante $\mathrm{o}$ mês $\mathrm{t}$; $\mathrm{P}_{\mathrm{rt}} \mathrm{O}$ volume precipitado no reservatório $\mathrm{r}$ durante o mês $t$; $\mathrm{Ev}_{\mathrm{rt}}$ o volume evaporado no reservatório $\mathrm{r}$ durante o mês $\mathrm{t}$ e $\mathrm{Qn}_{\mathrm{ct}} \mathrm{o}$ volume afluente ao reservatório $\mathrm{r}$ oriundo de contribuições da c- ézima calha (trecho) do rio a montante do reservatório $\mathrm{r}$ no mês $\mathrm{t}$.

O volume precipitado e evaporado no reservatório $\mathrm{r}$ em cada mês t é dado pelas equações:

$\mathrm{P}_{\mathrm{rt}}=\mathrm{p}_{\mathrm{rt}}\left(\frac{\mathrm{AR}_{\mathrm{rt}}+\mathrm{AR}_{\mathrm{r}(\mathrm{t}-1)}}{2}\right)$

$\mathrm{E}_{\mathrm{rt}}=\mathrm{e}_{\mathrm{rt}}\left(\frac{\mathrm{AR}_{\mathrm{rt}}+\mathrm{AR}_{\mathrm{r}(\mathrm{t}-1)}}{2}\right)$

sendo $p_{\mathrm{rt}}$ a taxa de precipitação para o reservatório $r$ no mês $t ; e_{\mathrm{rt}}$ a taxa de evaporação para o reservatório $\mathrm{r}$ no mês $\mathrm{t} ; \mathrm{AR}_{\mathrm{rt}}$ a área do espelho d'água do reservatório $\mathrm{r}$ no final do mês $\mathrm{t} ; \mathrm{AR}_{\mathrm{r}(\mathrm{t}-1)} \mathrm{a}$ área do espelho d'água do reservatório $\mathrm{r}$ no inicio do mês t.

A área do espelho de água do reservatório é relacionada com o volume do reservatório através de segmentos de reta na curva área-volume de modo a obter um bom ajuste linear da mesma, como exemplificado na Figura 5.

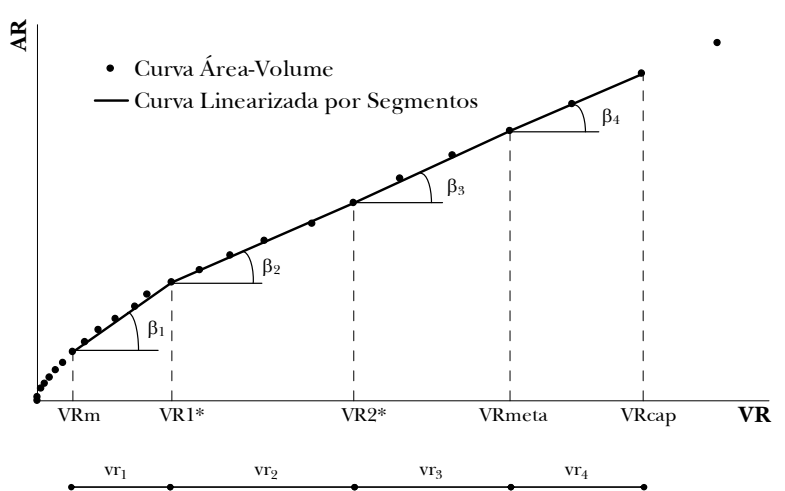

Figura 5 - Exemplo de um gráfico da curva área-volume linearizada de um reservatório $r$.

Assim a área do espelho d'água do reservatório r no mês t $\left(\mathrm{AR}_{\mathrm{rt}}\right)$ será dada pela expressão:

$\mathrm{AR}_{\mathrm{rt}}=\mathrm{ARm}_{\mathrm{r}}+\sum_{\mathrm{m}=1}^{\mathrm{nm}} \beta_{\mathrm{mr}} \cdot \mathrm{vr}_{\mathrm{mrt}}$

sendo, $\mathrm{ARm}_{\mathrm{r}}$ a área do espelho de água do reservatório $r$ correspondente ao volume morto do mesmo; $\beta_{\mathrm{mr}}$ coeficiente angular do segmento de reta $\mathrm{m}$ da curva área-volume do reservatório $\mathrm{r}$.

Os volumes de controle (ou pontos de quebra) utilizados devem ser os mesmos considerados 
na linearização da Equação 6. Desse modo as Equações 7 e 22 terão os mesmos trechos segmentados $m$ do volume de água do reservatório $\mathrm{r}\left(\mathrm{vr}_{\mathrm{mrt}}\right)$.

O volume a ser captado através das tomadas d'água está limitado pela sua capacidade máxima $\left(\right.$ Qtmax $\left._{\mathrm{d}}\right)$ e a cota de entrada do tubo da tomada d'água d no reservatório $\mathrm{r}\left(\mathrm{Ht}_{\mathrm{d}(\mathrm{r})}\right)$, escrito matematicamente pela expressão:

$0 \leq \mathrm{Qt}_{\mathrm{dt}} \leq \mathrm{Qtmax}_{\mathrm{d}}$

$\mathrm{Qt}_{\mathrm{d}(\mathrm{r}) \mathrm{t}} \geq 0$, se $\mathrm{HR}_{\mathrm{rt}} \geq \mathrm{Htr}_{\mathrm{d}(\mathrm{r})}$

sendo $\mathrm{HR}_{\mathrm{rt}}$ a cota do nível d'água do reservatório $\mathrm{r}$ no mês t.

Os volumes extravasados através dos vertedouros são limitados de acordo com as equações abaixo:

$0 \leq \mathrm{Qv}_{\mathrm{rt}} \leq \mathrm{Qvmax}_{\mathrm{r}}$

$\mathrm{Qv}_{\mathrm{rt}} \geq 0$, se $\mathrm{HR}_{\mathrm{rt}} \geq$ Hvert $_{\mathrm{r}}$

sendo Qvmax ${ }_{\mathrm{r}}$ o volume mensal máximo vertido que é projetado para o reservatório $r$; Hvert representa a cota da soleira do vertedor do reservatório $r$.

O volume liberado através da descarga de fundo $\left(\mathrm{Qf}_{\mathrm{rt}}\right)$ do reservatório $\mathrm{r}$ em cada mês t está limitado pela cota de entrada da tubulação de descarga de fundo $\left(\mathrm{Hfe}_{\mathrm{r}}\right.$ ) e pelo volume máximo que pode ser aduzida pela descarga de fundo em cada mês $t\left(\mathrm{Qfmax}_{\mathrm{rt}}\right)$, matematicamente temos:

$0 \leq \mathrm{Qf}_{\mathrm{rt}} \leq \mathrm{Qfmax}_{\mathrm{rt}}$

$\mathrm{Qf}_{\mathrm{rt}} \geq 0$, se $\mathrm{HR}_{\mathrm{rt}} \geq \mathrm{Hfe}_{\mathrm{r}}$

O volume máximo que pode ser aduzido pela descarga de fundo $\left(\mathrm{Qfmax}_{\mathrm{rt}}\right)$ pode ser estimado pela equação abaixo, referida em DAEE (2005):

Qf $\max _{\mathrm{rt}}=\mathrm{Cf}_{\mathrm{r}} \cdot \mathrm{Af}_{\mathrm{r}} \cdot \sqrt{2 \mathrm{~g}\left(\mathrm{HR}_{\mathrm{rt}}-\mathrm{Hfs}_{\mathrm{r}}\right)}$

sendo $\mathrm{Cf}_{\mathrm{r}}$ o coeficiente de vazão de descarga de fundo do reservatório $\mathrm{r} ; \mathrm{Af}_{\mathrm{r}}$ a área da seção transversal do tubo de descarga de fundo do reservatório $\mathrm{r}$ e $\mathrm{Hfs}_{\mathrm{r}}$ a cota de jusante da geratriz inferior do tubo de descarga de fundo do reservatório $\mathrm{r}$.

A curva da vazão máxima aduzida por descarga de fundo em função do volume de água do reservatório é obtida com o auxilio da curva cotavolume sendo a mesma linearizada através de segmentos de reta, como exemplificado na Figura 6, na qual VR1* representa o volume correspondente a cota de entrada da tubulação de descarga de fundo $\left(\mathrm{Hfe}_{\mathrm{r}}\right.$ ). Os volumes de controle (ou pontos de quebra) devem ser os mesmos que foram utilizados para a linearização da Equação 6.

A vazão máxima aduzida pela descarga de fundo do reservatório $r$ será dada pela expressão:

Qf $\max _{\mathrm{rt}}=\sum_{\mathrm{m}=1}^{\mathrm{nm}} \gamma_{\mathrm{mr}} * \mathrm{vr}_{\mathrm{mrt}}$

sendo $\gamma_{\mathrm{mr}}$ coeficiente angular do segmento de reta m da curva da vazão máxima aduzida pela descarga de fundo versus o volume de água do reservatório $\mathrm{r}$.

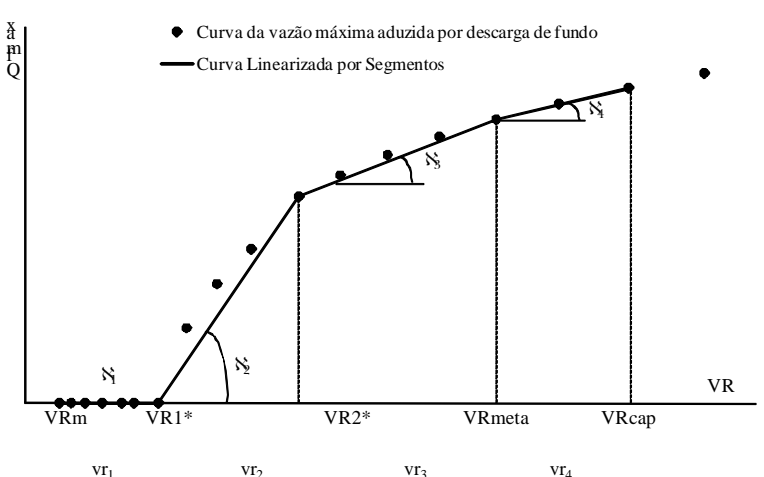

Figura 6 - Exemplo de um gráfico da vazão máxima aduzida por descarga de fundo versus o volume de água do reservatório $r$.

Na operação do reservatório, tem-se um volume final $\left(\mathrm{VR}_{\mathrm{t}}\right)$ e um volume inicial $\left(\mathrm{VR}_{(\mathrm{t}-1)}\right)$ que resultarão em duas vazões máximas $\left(\mathrm{Qfmax}_{\mathrm{t}} \mathrm{e}\right.$ Qf$\max _{(t-1)}$ ) que poderão ser aduzidas pela descarga de fundo para um mês t. Para minimizar esse problema utilizou-se média destas duas vazões.

$\mathrm{O}$ volume de água do reservatório $\mathrm{r}$ esta limitado por:

$\mathrm{VRm}_{\mathrm{r}} \leq \mathrm{VR}_{\mathrm{rt}} \leq \mathrm{VRcap}_{\mathrm{r}}$

A sustentabilidade hídrica do sistema pode ser garantida fazendo-se que o volume de água do reservatório no último mês de estudo seja igual ou superior ao volume inicial estabelecido para o mesmo. (Curi e Curi, 2001).

Com relação à agricultura irrigada as restrições físicas consideradas são as áreas a serem irrigadas em cada perímetro irrigado, a vazão a ser capta- 
da para o perímetro irrigado e a não negatividade das variáveis de decisão.

As limitações impostas pelas capacidades dos canais ou dos sistemas adutores, pelos quais a água será transportada até os respectivos perímetros irrigados, podem ser representadas por:

$$
\sum_{\mathrm{j}=1}^{\mathrm{nc}} \mathrm{Qirr}_{\mathrm{kjt}} \cdot \mathrm{Ac}_{\mathrm{kjn}} \leq \mathrm{Qt} \max _{\mathrm{kt}}
$$

sendo Qtmax ${ }_{\mathrm{kt}}$ a capacidade de vazão do sistema de captação para o perímetro irrigado k no mês t.

As restrições quanto ao limite máximo mensal de área que poderá ser plantada por perímetro em cada mês, pode ser obtida pela expressão:

$$
\sum_{j=1}^{n c} \xi_{k j t} \cdot A c_{j k n} \leq A p \max _{k t}
$$

sendo $\operatorname{Apmax}_{\mathrm{kt}}$ o limite máximo da área total que poderá ser plantada no perímetro irrigado k no mês t. Sendo:

$\xi_{\mathrm{kjt}}=1$, se a cultura agrícola $\mathrm{j}$ for plantada no mês $\mathrm{t}$ no perímetro irrigado $\mathrm{k}$;

$\xi_{\mathrm{kjt}}=0$, se a cultura agrícola $\mathrm{j}$ não for plantada no mês t no perímetro irrigado $\mathrm{k}$.

Com relação aos critérios agronômicos e de mercado, o conjunto de restrições se refere aos limites mínimos e máximos das áreas a serem plantadas com cada tipo de cultura plantada por perímetro irrigado, expressa por:

$\operatorname{Acmin}_{\mathrm{kjn}} \leq \mathrm{Ac}_{\mathrm{kjn}} \leq \mathrm{Acmax}_{\mathrm{kjn}}$

sendo Acmin $_{\text {kjn }}$ a área mínima a ser plantada com a cultura agrícola j no perímetro irrigado k no ano n; $A_{c m a x}$ kjn área máxima a ser plantada com a cultura agrícola j no perímetro irrigado $\mathrm{k}$ no ano $\mathrm{n}$.

Dentre os componentes do sistema hídrico estão as calhas dos rios cujas vazões podem estar limitadas por valores inferiores, indicando requerimentos de regularizações e de vazões ecológicas para saneamento do rio, ou superiores, para o controle de cheias, que podem ser descritas matematicamente por:

$\operatorname{Qnmin}_{\mathrm{ct}} \leq \mathrm{Qn}_{\mathrm{ct}} \leq \mathrm{Qnmax}_{\mathrm{ct}}$

sendo Qnmin ${ }_{\mathrm{ct}}$ a vazão mínima na c-ézima calha de rio no mês t; Qnmax ${ }_{\mathrm{ct}}$ é a vazão máxima na c-ézima calha de rio no mês t.
Além destas restrições, o modelo promove, ainda, o balanço hídrico em cada nó do sistema s, através da seguinte expressão:

$\sum_{\mathrm{e}}$ Qentra $_{\text {est }}=\sum_{\mathrm{i}} \mathrm{Qsai}_{\text {ist }}$

sendo Qentra ${ }_{\text {ent }}$ a e-ézima vazão de entrada no nó s, no mês t; Qsai ${ }_{\text {ist }}$ a i-ézima vazão de saída do nó s, no mês t.

As perdas por evaporação e a infiltração nos segmentos das calhas dos rios são avaliadas através de coeficientes de perda, que devem ser especificados para os segmentos e representam as frações dos fluxos que seriam perdidos durante o percurso nos segmentos das calhas dos rios.

\section{Processo iterativo de otimização e análise de convergência}

O Artifício de Linearização por Segmentos garante para a minimização de uma função convexa (caso da Equação 6) que os trechos segmentados da variável de decisão serão "percorridos" numa forma seqüencial, do primeiro ao último, atingindo a cada trecho percorrido o seu valor máximo. Porém, observou-se que, em certos casos, isso não ocorre. Para a modelagem proposta, pode haver combinações dos valores dos trechos segmentados das relações cota $\mathrm{x}$ área $\mathrm{x}$ volume para os volumes de água armazenados no reservatório em um mês t que resultem em um aumento do atendimento (de forma incorreta) a uma demanda ou restrição imposta. Isso ocorre devido ao caráter multiobjetivo do modelo, em que o atendimento de uma determinada demanda do sistema pode ter maior prioridade do que o volume meta do reservatório. Assim, por exemplo, quando o sistema estiver em um período crítico (como um ano seco ou no atendimento de uma demanda muito alta, por exemplo), para se garantir o atendimento das demandas com prioridade maior do que o volume meta do reservatório, o volume evaporado pode ser diminuído preenchendo os últimos trechos segmentados do volume de água armazenado no reservatório, para curva área-volume convexa. Neste caso, as declividades dos últimos trechos segmentados são menores do que nos primeiros trechos, o que resultará em um menor valor da área do espelho d'água do reservatório e, conseqüentemente, no volume evaporado, garantindo assim uma maior quantidade de água para o suprimento das demandas.

Para contornar tal problema é utilizado um procedimento iterativo no uso da PL, denominado 
Programação Linear Seqüencial (ou Sucessiva) onde a cada iteração os trechos segmentados do volume do reservatório são ajustados de modo a resultarem em um volume de água armazenado de forma correta.

Para melhor explicar o procedimento, vamos supor que o volume meta de um reservatório em um mês qualquer seja 7,5, logo a função objetivo será:

$\mathrm{VM}=\left(\frac{7,5-\mathrm{VR}}{7,5}\right)$

O volume de água do reservatório (VR) e a função objetivo linearizada (VM') poderão ser obtidos através das expressões:

$\mathrm{VR}=0,5+\mathrm{vr} 1+\mathrm{vr} 2+\mathrm{vr} 3+\mathrm{vr} 4$

$$
\begin{aligned}
\mathrm{VM}^{\prime} & =0,87-0,23 \cdot \mathrm{vr}_{1}-0,16 \cdot \mathrm{vr}_{2} \\
& -0,06 \cdot \mathrm{vr}_{3}+0,05 \cdot \mathrm{vr}_{4}
\end{aligned}
$$

com $0 \leq \mathrm{vr}_{1} \leq 1,25 ; 0 \leq \mathrm{vr}_{2} \leq 2,25 ; 0 \leq \mathrm{vr}_{3} \leq 3,50$ e $0 \leq$ $\mathrm{vr}_{4} \leq 3,00$.

Supondo que ao resolver o problema de otimização pela primeira vez ( $1^{\mathrm{a}}$ iteração) com todas as restrições impostas, o volume de água do reservatório VR seja igual a 6,0. Porém os valores dos trechos segmentados foram: $\mathrm{vr}_{1}=0 ; \mathrm{vr}_{2}=0 ; \mathrm{vr}_{3}=2,50$; vr4 $=3,00$ (os valores corretos seriam: $\mathrm{vr} 1=1,25$; vr2 $=2,25 ; \operatorname{vr} 3=2,00 ; \operatorname{vr} 4=0)$.

Os valores incorretos dos trechos segmentados resultam em valores incorretos da área do espelho de água do reservatório e da vazão máxima que pode ser aduzida pela descarga de fundo, pois ambas as curvas são linearizadas com os mesmos trechos segmentados utilizados na linearização da função objetivo do volume meta do reservatório.

$\mathrm{O}$ ajuste proposto para resolver tal incoerência consiste em impor restrições, inicialmente com certa relaxação nos valores dos trechos segmentados, para que, na próxima iteração, o valor do volume de água armazenado no reservatório seja próximo ao que foi obtido na iteração anterior

Para o exemplo proposto, inicialmente será estabelecido que: $\mathrm{vr}_{1}=1,25$ (seu valor máximo) e $\mathrm{vr}_{4}$ $=0$. Os trechos segmentados $\mathrm{vr}_{2}, \mathrm{vr}_{3}$ continuam com suas restrições iniciais. Aplicando-se a PL novamente - 2 ${ }^{\mathrm{a}}$ iteração - podemos obter os seguintes valores: $\mathrm{vr}_{1}$ $=1,25 ; \mathrm{vr}_{2}=0,45 ; \mathrm{vr}_{3}=3,5 ; \mathrm{vr}_{4}=0 ;$ resultando num volume de água do reservatório, ainda incorreto, de $\mathrm{VR}=5,7$.
A Figura 7 mostra os valores do volume de água do reservatório entre a $1^{\underline{a}}$ e a $2^{\underline{a}}$ iteração e a restrição imposta aos trechos segmentados para a $2^{\underline{a}}$ iteração. Nota-se que, tanto na $1^{\underline{a}}$ iteração como na $2^{a}$ iteração, o valor do volume de água do reservatório permaneceu entre os valores de 4,00 e 7,50. Espera-se que o valor ótimo do volume de água do reservatório esteja entre esses valores. Assim, na próxima iteração - 3 $3^{\mathbf{a}}$ iteração - será estabelecido que: $\mathrm{vr}_{1}=1,25 ; \mathrm{vr}_{2}=2,25$ (seus valores máximos) $\mathrm{e}$ $\mathrm{vr}_{4}=0$. O trecho segmentado $\mathrm{vr}_{3}$ continuará com sua restrição inicial.

Logo, qualquer que seja o valor estabelecido para o trecho segmentado $\mathrm{vr}_{3}$, o valor do volume de água do reservatório VR, e, conseqüentemente, da função objetivo VM', da área do espelho de água do reservatório e da vazão de descarga de fundo máxima serão determinados de forma correta, ou seja, qualquer valor que seja estabelecido no trecho segmentado $\mathrm{vr}_{3}$, os seus trechos anteriores $\left(\mathrm{vr}_{1} \mathrm{e} \mathrm{vr}_{2}\right)$ estão com seus valores máximos e seus trechos posteriores $\left(\mathrm{vr}_{4}\right)$ são nulos.

\section{$1^{\underline{a} \text { iteração }}$}
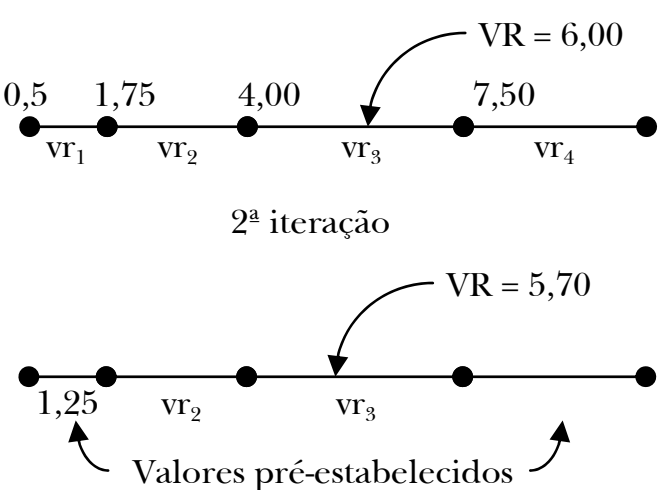

Figura 7 - Valor do volume de água do reservatório e a representação restrição imposta aos trechos segmentados para a $2^{\circ}$ iteração.

Tem-se, na maioria das vezes, que logo na primeira iteração os valores de cada trecho segmentado podem estar corretos, indicando assim a solução ótima do problema. Porém, quando isso não ocorre, para uma boa aceitação dos resultados, além da verificação dos valores dos trechos segmentados, é analisado o processo de convergência da função objetivo fo, que é avaliado através do cálculo do erro relativo $\left(\operatorname{erro}_{\mathrm{fo}}\right)$, dado por: 


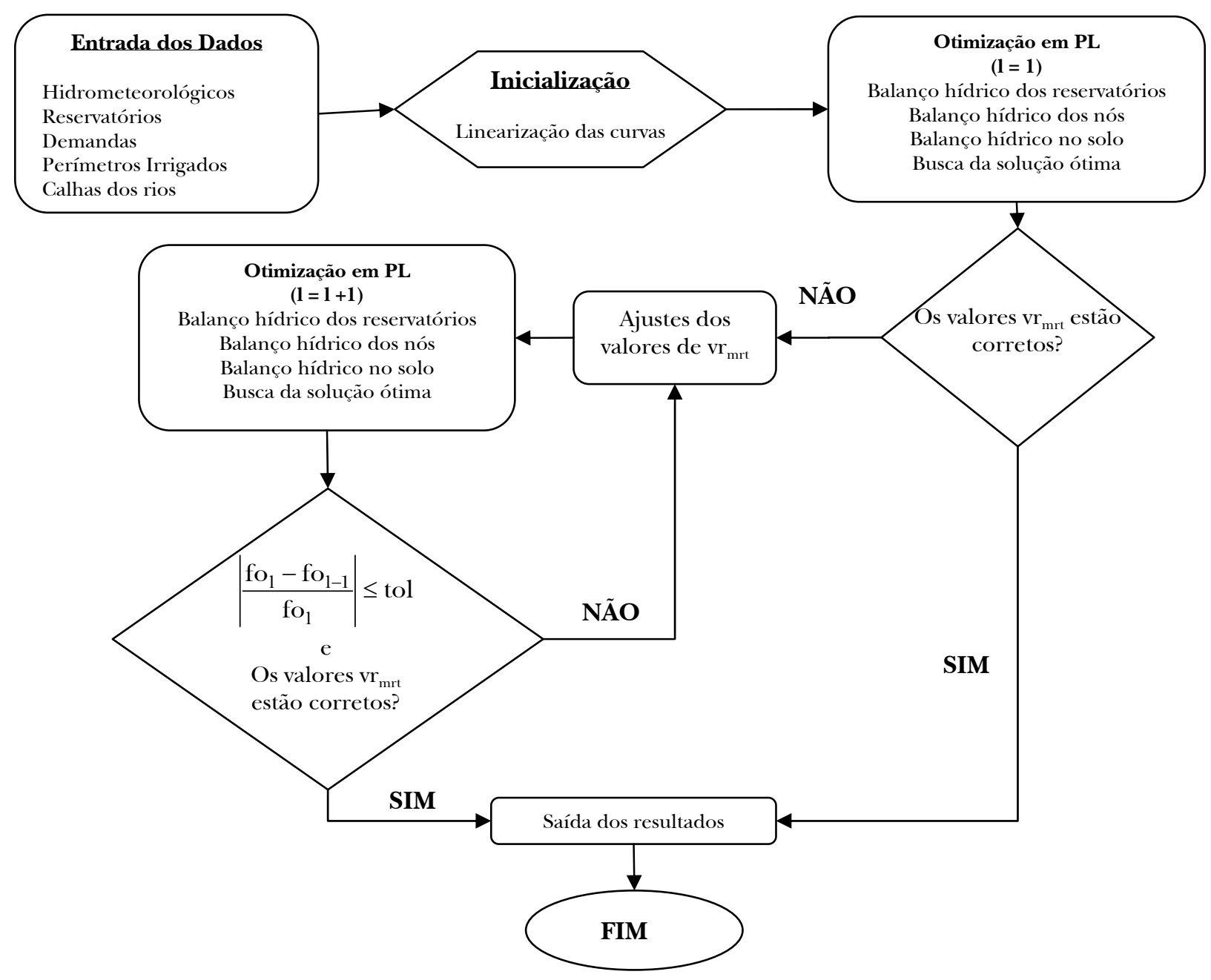

Figura 8 - Fluxograma do modelo de otimização.

$\operatorname{erro}_{f o}=\left|\frac{f_{1}-f o_{1-1}}{f o_{1}}\right| \leq$ tol

onde, fo $_{1}$ é o valor da função objetivo para a l-ézima iteração $(1 \geq 2)$; tol é o nível de tolerância especificado para o sistema a ser otimizado.

O fluxograma geral do modelo está apresentado na Figura 8.

\section{CONCLUSÃO}

O trabalho apresentou o desenvolvimento de um modelo de otimização multiobjetivo para a operação integrada de reservatórios, rios e áreas irrigadas, utilizando a programação linear. O modelo foi concebido em termos de componentes prédefinidos como reservatórios, calhas de rios, demandas mensais de água, perímetros irrigados, sistemas de bombeamento, culturas agrícolas e sistemas de irrigação. As funções objetivo e restrições conferem ao modelo o caráter desejado da generalidade de sua aplicação, ou seja, facilidade de estabelecimento de uma variedade de cenários climáticos, operacionais, agronômicos, ambientais, etc. As nãolinearidades foram representadas por segmentos de retas (através do Artifício de Linearização por Segmentos) e a solução do problema fez uso de um processo iterativo (Programação Linear Seqüencial) até atingir uma convergência aceitável e prédefinida. 
O método proposto para a implementação das não-linearidades dos componentes do sistema, representadas nas equações de restrições do problema e das funções objetivo, permite, entre outras coisas, o uso da PL com todas as suas vantagens, consistência nos valores obtidos por causa da convergência numérica e um tempo de processamento satisfatório para a obtenção de uma solução ótima.

O modelo torna-se bastante útil para aplicação em regiões de escassos recursos hídricos, como o semi-árido brasileiro, nas quais perímetros de irrigação de pequeno a médio porte são, em geral, alimentados por reservatórios de pequeno e médio portes e, portanto, mais sujeitos aos efeitos de variações climáticas. Advém daí, portanto, a extrema importância do planejamento e otimização conjunta da definição das áreas irrigadas e do uso da água do reservatório de forma a que o produtor não venha a ter sérios prejuízos em função do não atendimento da demanda hídrica das suas culturas irrigadas, principalmente as perenes.

\section{AGRADECIMENTOS}

Ao CTHIDRO/CNPq pelo o apoio concedido ao primeiro autor.

\section{REFERÊNCIAS}

AHMED, J. A.; SARMA, A. K. (2005) Genetic Algorithm for Optimal Operating Policy of a Multipurpose Reservoir. In: Water Resources Management 19: 145-161

BARBOSA, P. S. F. (2002) Modelos de Programação Linear em Recursos Hídricos. In: Técnicas Quantitativas para o Gerenciamento de Recursos Hídricos. $2^{\mathrm{a}}$ Edição. Editora da Universidade/UFRGS-ABRH. Porto Alegre, p. 97-163.

BRAGA, B.; GOBETTI, L. (2002). Analise Multiobjetivo. In: Técnicas Quantitativas para 0 Gerenciamento de Recursos Hídricos. $2^{\mathrm{a}}$ Edição. Editora da Universidade/UFRGS-ABRH. Porto Alegre, p. 361-420.

CURI, R. C.; CURI, W. F.; OLIVEIRA, M. B. (2004). Analise de Alterações na Receita Líquida de um Perímetro Irrigado no Semi-árido sob condições de Variações Hídricas e Econômicas. In: Revista Brasileira de Recursos Hídricos 9(3): 39-53.

CURI, W. F.; CURI, R.C. (2001). ORNAP - Optimal Reservoir Network Analysis Program. In: Anais do XIV Simpó- sio Brasileiro de Recursos Hídricos. Aracaju. CDROM

DAEE (2005). Guia Pratico para Projetos de Pequenas Obras Hidráulicas. São Paulo. Secretaria de Estado de Energia, Recursos Hídricos e Saneamento. Departamento de Águas e Energia Elétrica. 116p.

DIAZ, G. E.; BROWN, T. C.; SVEINSSON, O. (2000) Aquarius: A Modeling System for River Basin Water Allocation. General Technical Report RM-GTR-299-revised, Fort Collins, CO: U.S. Department of Agriculture, Forest Service, Rocky Mountain Forest and Range Experiment Station, $172 \mathrm{p}$.

HSU, N.-S.; CHENG, W.-C.; CHENG, W.-M.; WEI, C.-C.; YEH, W. W.-G. (2008) Optimization and Capacity Expansion of a Water Distribution System. In: Advances in Water Resources 31: 776-786.

LANNA, A.E. (1998). Nota de Aula: Cap. 5. Tópicos Avançados em PL: Método Simplex Revisado, Análise PósÓtimo e Artifícios de Linearização. Disciplina: Análise de Sistêmica de Recursos Hídricos. Programa de Pós-Graduação em Recursos Hídricos e Saneamento da UFRGS. p. 75-87.

MOUSAVI, S. J.; MOGHADDAM, K. S.; SEIFI, A. (2004) Application of an Interior-Point Algorithm for Optimization of a Large-Scale Reservoir System. In: Water Resources Management 18: 519-540

SUDHA, V.; VENUGOPAL K.; AMBUJA, N. K. (2008). Reservoir Operation Management through Optimization and Deficit Irrigation. In: Irrigation Drainage System 22: 93-102.

\section{A Multiobjective Optimization Model for Water Resource System Analysis: II Application}

\section{ABSTRACT}

This work involves the application of a multiobjective optimization model, based on sequential linear programming, to a two-reservoir system connected in series, and $a 500$ ha irrigated perimeter in the semiarid region of Paraiba state. The water demands considered include urban water supply, irrigation and river flow regulation downstream from the reservoirs. The following objectives were considered to establish an optimal operation: to meet urban water supply demands, to regulate flow downstream from the reservoirs and establish target reservoir volumes of reservoir, as well as maximizing the labor required in agriculture and net profit. Scenarios with various priorities for different objects were created to evaluate the model response regarding to the consistency of results and required computation time. The results showed that the objectives were fulfilled in accordance with the assigned priorities and the 
Um Modelo de Otimização Multiobjetivo para Análise de Sistema de Recursos Hídricos I: Metodologia

problem constraints were met. The computation time and the number of iterations can be considered satisfactory (19 seconds and 5 iterations, in average, for a problem having 3802 decision variables and 874 constraints when considering 9 year of system operation). The attained system operational results can provide support to the generation of operational rules for water allocation in order to meet the required demands, objectives and physical constraints.

Key-words: Water Resource, optimization, linear programming, linearization. 